\title{
Video Article \\ Proton Therapy Delivery and Its Clinical Application in Select Solid Tumor Malignancies
}

\author{
Adeel Kaiser ${ }^{1}$, John G. Eley ${ }^{1}$, Nasarachi E. Onyeuku ${ }^{1}$, Stephanie R. Rice ${ }^{1}$, Carleen C. Wright ${ }^{2}$, Nathan E. McGovern ${ }^{2}$, Megan Sank ${ }^{2}$, \\ Mingyao Zhu ${ }^{1}$, Zeljko Vujaskovic ${ }^{1}$, Charles B. Simone $2 \mathrm{nd}^{1}$, Arif Hussain ${ }^{3}$ \\ ${ }^{1}$ Department of Radiation Oncology, University of Maryland School of Medicine \\ ${ }^{2}$ Department of Radiation Oncology, University of Maryland Medical Center \\ ${ }^{3}$ Department of Medicine, University of Maryland School of Medicine, University of Maryland Greenebaum Comprehensive Cancer Center
}

Correspondence to: Adeel Kaiser at akaiser@som.umaryland.edu

URL: https://www.jove.com/video/58372

DOI: doi: $10.3791 / 58372$

Keywords: Medicine, Issue 144, Pencil beam scanning protons, passive scatter protons, intensity modulated proton therapy, proton therapy, particle therapy, prostate cancer

Date Published: 2/6/2019

Citation: Kaiser, A., Eley, J.G., Onyeuku, N.E., Rice, S.R., Wright, C.C., McGovern, N.E., Sank, M., Zhu, M., Vujaskovic, Z., Simone 2nd, C.B., Hussain, A. Proton Therapy Delivery and Its Clinical Application in Select Solid Tumor Malignancies. J. Vis. Exp. (144), e58372, doi:10.3791/58372 (2019).

\section{Abstract}

Radiation therapy is a frequently used modality for the treatment of solid cancers. Although the mechanisms of cell kill are similar for all forms of radiation, the in vivo properties of photon and proton beams differ greatly and maybe exploited to optimize clinical outcomes. In particular, proton particles lose energy in a predictable manner as they pass through the body. This property is used clinically to control the depth at which the proton beam is terminated, and to limit radiation dose beyond the target region. This strategy can allow for substantial reductions in radiation dose to normal tissues located just beyond a tumor target. However, the degradation of proton energy in the body remains highly sensitive to tissue density. As a consequence, any changes in tissue density during the course of treatment may significantly alter proton dosimetry. Such changes may occur through alterations in body weight, respiration, or bowel filling/gas, and may result in unfavorable dose deposition. In this manuscript, we provide a detailed method for the delivery of proton therapy using both passive scatter and pencil beam scanning techniques for prostate cancer. Although the described procedure directly pertains to prostate cancer patients, the method may be adapted and applied for the treatment of virtually all solid tumors. Our aim is to equip readers with a better understanding of proton therapy delivery and outcomes in order to facilitate the appropriate integration of this modality during cancer therapy.

\section{Video Link}

The video component of this article can be found at https://www.jove.com/video/58372/

\section{Introduction}

It is estimated that 1.7 million individuals in the United States will be diagnosed with cancer in 2018 , with over 600,000 succumbing to the disease $^{1}$. Current treatment options involve mono- or multi-modality therapy using surgery, radiation therapy (RT), and systemic treatments. With respect to $\mathrm{RT}$, one-quarter of newly diagnosed patients will receive it as part of their initial cancer therapy and almost half will ultimately require it during their disease course ${ }^{2,3}$.

The advent of RT dates back to 1895 when William Conrad Roentgen discovered X-ray while working with a cathode-ray tube in his laboratory at Würzberg University in Germany ${ }^{4}$. Not long after, patients with wide ranging diseases such as lupus and cancer were receiving treatments using radium rays. Early complications were quickly realized and were even discussed by Pierre Curie in his Nobel Prize lecture 5 . Since radiation affects both normal and tumor tissues, carefully controlled doses of radiation must be utilized to maximize the therapeutic ratio, defined as the probability of tumor control versus the probability of unacceptable toxicity. With gradual advancements in technology, as well as better understanding of radiobiology and physics, this therapeutic ratio has greatly improved with time. The use of RT has significantly enhanced outcomes for several cancers, as reflected by its inclusion in national guidelines for cancer therapy ${ }^{6,7,8,9}$. In some cases, RT may be used as the sole modality for therapy ${ }^{10}$, whereas in other diseases, it may be used as part of multi-modality therapy for local disease control or the eradication of microscopic disease ${ }^{11}$. Although often used with a curative intent, many RT patients are treated for palliation of pain or other symptoms that develop from tumor-induced compression, invasion, or destruction of normal tissues in the setting of loco-regional or widespread, metastatic disease.

The basic principles behind RT are straightforward. With the application of radiation, energy is deposited into cells through the ionization of atoms. This energy, though it may raise the temperature of an irradiated region by only a few microkelvin, produces free radicals that can directly damage exposed cells by way of DNA injury ${ }^{12,13}$. Much of our understanding of high-energy particle radiation and its interaction with matter comes from theoretical and experimental studies of cosmic rays and their interactions in the upper atmosphere carried out in the early $20^{\text {th }}$ century $^{14}$. High-energy ( $\mathrm{MeV}$ to $\mathrm{GeV}$ ) charged particles interact with matter primarily via the electromagnetic force: as these particles pass 
through matter or tissue, inelastic collisions with orbital electrons lead to ionization and excitation of target matter, and elastic collisions with atomic nuclei lead to scatter or deflection of the particle path. In addition, nuclear collisions and hard collisions with electrons lead to a cascade of secondary radiation that adds to the ionizing effect of particle radiation. High-energy particles traversing matter thus leave behind a wake of ionized atoms, molecules, and free electrons that are chemically reactive and can potentially induce biologic changes or damage to organisms exposed to these ionizing fields.

A major long-term goal of radiotherapy has been to learn how best to harness these ionizing fields in a manner that will effectively treat human disease. Clinically, the ideal form of radiation (such as photon, proton, electron, or heavy ion) should induce sufficient ionization in the disease target to provide therapeutic anti-tumor effect, while at the same time cause minimal ionization in the surrounding normal tissues to minimize deleterious effects. Which type of radiation is selected for RT depends in part on the disease being treated. For tumors that are located deeper within the body and may also be surgically inoperable, megavolt photons, protons, and heavy ions are considered optimal ${ }^{15,16}$. For superficial cancers, such as those involving the skin, electron therapy may be optimal and even preferable to surgery for cosmesis. On the other hand, the advantage of megavolt photons lies in their ability to penetrate deep in the tissue while limiting damage to the skin. In the case of charged particles, such as electrons, protons, or heavy ions, their primary advantage lies in their 'stopping' characteristics; that is, charged particles lose energy continuously via the inelastic collisions described above, and this energy loss is highly predictable on the millimeter scale. Therefore, a charged particle beam can be delivered to a patient with precise energies to desired depths. Further, charged particles produce little to no exit dose $^{17}$. By contrast, uncharged particles like photons exhibit an exponential falloff (attenuation) with increasing depth, which often leads to a significant exit dose that may compromise healthy tissues distal to the target. These concepts are demonstrated in Figure 1, which shows the radiation dose (ionization) properties of the various types of radiation used clinically. A central motivation for using protons or carbon ions instead of photons for deeper tumor targets is that there is minimal dose entry dose and near zero exit dose beyond target tissues. Table 1 summarizes some of the clinically relevant characteristics of photon and proton beams.

Advances in the field of radiotherapy, including proton therapy, have occurred on two major fronts: 1) the building of efficient particle accelerators capable of producing high-energy $(\mathrm{MeV})$ radiation such as synchrotron and cyclotron accelerators, and 2) the development of sophisticated computational methods that combine disease imaging data and radiation transport calculations to allow computer-simulated "treatment planning." For treatment planning, patients typically undergo computed tomography (CT) imaging. The CT images contain 3-dimensional anatomic information about the patient as well as precise quantification of the tissue densities. The CT images and density maps are then used in computer simulations to plan the radiation treatment: both the energy and intensity of the radiation field are mathematically optimized for each patient. A magnetic resonance imaging (MRI) scan or a positron-emission tomography (PET) scan may also be used to complement the CT data.

Below, we describe a step-by-step outline of how patients are navigated through their radiation treatment course, followed by the examples of certain tumor types treated with proton therapy.

\section{Protocol}

The exact radiation therapy planning and delivery protocol will vary by disease site and may even require individualization for each patient. Moreover, the procedure may also require modifications to accommodate institutional preferences and equipment availability. For the purpose of this discussion, we will outline the steps used for proton planning in a typical prostate cancer case since this is the most common cancer treated with proton therapy at U.S. centers ${ }^{18}$. This protocol describes standard-of-care clinical procedures, and so does not require institutional approval by human research ethics committee.

\section{Computed Tomography Simulation for Radiation Treatment}

1. Insert the extended tabletop into the treatment table and ensure that it is locked.

2. Place an inflated immobilization cushion over the table top and indexing bar for the leg mold at the level of the patient's knee.

3. Place a solid head rest at the top of the table. Start with F size head, which accommodates most patients.

4. Confirm that the patient has completed the full bladder process by drinking $16-24$ ounces of fluid 45 min prior to the scheduled scan time. This step needs to be completed prior to Step 1.12.

5. Register the patient into CT Patient Registration system.

6. Select prostate scanning protocol with the slice thickness of $3 \mathrm{~mm}$.

7. Confirm that the treatment consent, contrast consent and simulation order have been completed by the attending physician.

8. Ask the patient to change into a gown and remove all clothing from the waist down.

9. Confirm the patient identification by verbally verifying the patient's name, date-of-birth, and procedure site.

10. Take a face photo of the patient.

11. Ask the patient to sit on the table and then assist the patient into a supine, head-first position.

12. Place the patient's legs into the inflated immobilization cushion and position the patient's arms on the chest by interlacing their fingers, giving them a blue ring, or applying straps around their arms.

13. Confirm the patient alignment with the laser system.

14. Connect the dual vacuum pump to the nozzle of the immobilization cushion.

15. Place the legs in the immobilization cushion so that the mold will envelop the side of the legs and also create a barrier between the legs. Ensure that the immobilization cushion is below the pelvis and extends past the feet.

16. Insert and inflate ab endorectal balloon if clinically indicated per treating physician

17. Perform anterior-posterior (AP) and lateral (LAT) scout kilovoltage X-ray images (topograms) to confirm that patient alignment is optimized. This includes the verification of straightness on the AP image and rotational alignment on the lateral image. Use both images to ensure that bowel gas is minimal. Positioning should all be corrected and confirmed with re-imaging. If bowel gas is present and excessive, excuse the patient to the restroom and restart the process from Step 1.15.

18. Once the patient is in the desired position, remove the air from the immobilization cushion using the $Q$-fix dual vacuum pump to form a solid mold around their legs and feet. 
19. Manually adjust the treatment table so that the laser crosshairs are at the level of the patient hips and at the midline of the hips and abdomen at the level of the hip joint. Specify the crosshair locations on the patient using a marking pen. Place the localization markers at the crosshairs to designate a starting point for verification simulation during radiation delivery.

20. Set CT scanning parameters to include the pelvic region from the L3 spine to the mid-femur.

21. Scan the patient using the prostate scanning protocol.

22. Confirm that the scan is acceptable for treatment planning. The bladder should be full, and the rectum should have minimal air or stool.

23. Export the digital scan file to the treatment planning software and notify dosimetry.

24. Label the patient immobilization cushion with the patient ID, treating physician name, and setup instructions prior to storage for subsequent use during therapy.

25. Proceed to Step 2.1 if the patient is undergoing pencil beam proton therapy. Skip to Step 3.1 if the patient will receive passive scatter proton therapy.

\section{Radiation Treatment Planning Using Pencil Beam Therapy}

1. Import the CT simulation data into the treatment planning software (TPS).

2. Use treatment planning system contouring tools to define all relevant geometric volumes based on acquired CT images. These structures include the bladder, rectum, large bowel, small bowel, femoral heads, outer body surface, fiducials, rectal spacer, and/or endorectal balloon.

3. Create an additional external contour, which includes the body, the treatment table, and any immobilization devices. Radiation dose will only be calculated within this contour.

4. Contour the first clinical target volume (CTV1) to include the prostate, seminal vesicles, and involved lymph nodes. The CTV1 will be prescribed 45.0 Gy (RBE). The CTV1 volume will have the appearance of a U-shaped structure on axial images. Small bowel, rectum, and bladder normal tissues will reside within the U-shaped target volume.

5. Contour the second clinical target volume (CTV2) to include the prostate and seminal vesicles. The CTV2 will be prescribed a total dose of 34.2 Gy (RBE).

6. Select three beams at gantry angles of 90,180 and 270 degrees for the treatment of the CTV1 volume. Select only the 90 and 270 degree beam angles for CTV2 treatment.

7. Design two geometric blocking structures (avoidance volumes).

1. Create a "mid-block" avoidance volume encompassing the normal tissues within the U-shaped CTV1 volume.

2. Block the inferior aspect of the posterior-anterior (180 degree) beam below the level of the top of the prostate using a "rectum-block".

8. Create an isotropic 7-mm expansion of the CTV1 to form a proton planning target volume, named pPTV1. Use the pPTV1 to define a spot placement volume such that the proton Bragg peaks (also referred to as proton "spots") are positioned by the planning optimizer to cover the CTV1.

9. As in Step 2.6, create a similar expansion of the CTV2 volume to form pPTV2, but use 8-mm expansion in the left-right direction and 5-mm expansions in both the anterior-posterior and superior-inferior directions.

10. To enable robust optimization, a feature that can account for setup uncertainties, range uncertainties, and the variable gas filling of the intestine, create two artificial ("overridden") CT data sets: the first has the entire small intestine, large intestine, and rectum overwritten to the density of air, and the second has those volumes overwritten to the density of muscle.

11. Prior to optimization, create a new treatment plan for the CTV1. Designate the proton treatment machine to be used for planning, assign the prescribed dose and fractionation of $45 \mathrm{~Gy}(\mathrm{RBE})$ in 25 fractions, and define the primary target to be the CTV1. Assign $100 \%$ of the prescribed dose to cover at least $98 \%$ of the CTV1 volume using all 3 beams (right lateral, left lateral and posterior-anterior).

12. Create another treatment plan for the CTV2 with 2 beam sets. First, assign $18 \mathrm{~Gy}$ (RBE) to be delivered in 10 fractions to the CTV2 using only the left lateral beam, and assign $16.2 \mathrm{~Gy}$ (RBE) to be delivered in 9 fractions to the CTV2 using only the right lateral beam. Assign $100 \%$ of the prescribed dose to cover $100 \%$ of the CTV2 volume.

13. Prepare for the optimization of the CTV1 plan

1. Assign the mid-block structure as a range margin for the lateral beams and assign the rectum-block structure as a range margin for the posterior-anterior beam.

2. Start the optimization with automatic (default) settings for energy layer spacing, spot spacing, and target margin.

3. Specify a maximum number of iterations of 40 , a maximum number of optimizations before "spot filtering" of 10 , and a minimum spot weight of 1.5 monitor units. Spot filtering removes proton spots with less than 1.5 monitor units, as there is a technical minimum limit on the number of protons that can be delivered by the treatment machine.

4. In the first round of optimization for the CTV1, designate the target as the pPTV1 to establish the grid of proton spot positions. The specified objectives are $45.5 \mathrm{~Gy}(\mathrm{RBE})$ (weight $=100$ ) to the pPTV1 and a dose falloff of 45 to $0 \mathrm{~Gy}(\mathrm{RBE})$ within a distance of $1 \mathrm{~cm}$ (weight $=2$ ).

5. Begin a second round of optimization for CTV1, by deleting the pPTV1 objectives above. Then resume optimization with the new objectives and objective weights. Specify these parameters as follows to develop an intensity modulated plan, also known as Multi-field Optimization (MFO).

1. For the external volume, set a dose falloff of 45 to $0 \mathrm{~Gy}$ (RBE) within a distance of $1 \mathrm{~cm}$ and with a weight of 2 .

2. For the CTV1 volume, set a minimum dose of $45 \mathrm{~Gy}$ (RBE) with a weight of 100 .

3. For the CTV1 volume, set a uniform dose of $45.5 \mathrm{~Gy}(\mathrm{RBE})$ and a weight of 100 . Set this objective as robust.

4. For the pPTV1 volume, set the maximum dose at 46 Gy (RBE) with a weight of 100 and set this objective as robust.

5. For the rectum volume, set a maximum dose of $45.8 \mathrm{~Gy}(\mathrm{RBE})$ with a weight of 50 . Set this objective as robust.

6. For the bladder volume, set a maximum dose of $45.8 \mathrm{~Gy}$ (RBE) with a weight of 50 . Set this objective as robust.

7. For the small bowel, set a maximum dose of 45.8 Gy (RBE) with a weight of 50 . Set this objective as robust.

8. For the large bowel, set a maximum dose of $45.8 \mathrm{~Gy}$ (RBE) with a weight of 50 . Set this objective as robust. 
6. In addition to the specific objectives, assign robustness settings to mitigate 5 -mm positional shifts, $3.5 \%$ range uncertainty, and incorporate the artificial ("overridden") CT data mentioned above to address bowel gas variability. Apply these robustness settings only to those objectives specified above as "robust".

14. Complete the optimization for the CTV1 plan and review the resulting optimized plan to ensure that the prescription goals have been achieved.

15. Prepare for optimization of the CTV2 plan

1. Complete the first optimization for the CTV2 plan using the pPTV2 to achieve a spot placement grid as the CTV1 plan.

2. Delete the pPTV2 objectives and resume the optimization with new objectives (percentages are with respect to the prescription for the CTV2). For the CTV2 plan, optimize these objectives for the left and right beam individually. This is known as Single Field Optimization (SFO) and the objectives for each beam are as follows.

1. For the external volume, set a dose falloff of $34.2 \mathrm{~Gy}(\mathrm{RBE})$ to $0 \mathrm{~Gy}(\mathrm{RBE})$ within a distance of $5 \mathrm{~mm}$ and a weight of 2.

2. For the CTV2 volume, set a minimum dose of $34.37 \mathrm{~Gy}(\mathrm{RBE})$ with a weight of 120 .

3. For the CTV2 volume, set a uniform dose of 34.54 Gy (RBE) with a weight of 100 . Set this objective as robust.

4. For the pPTV2 volume, set a maximum dose of 34.88 Gy (RBE) with a weight of 100 . Set this objective as robust.

3. Use the same robustness settings for the optimization.

16. Complete the optimization separately for the left and right lateral beams to create 2 plan beam sets. This will permit the delivery of radiation to CTV2 using either the left or right lateral beams. This is in contrast to CTV1 dose delivery, which requires all 3 beams (RL, LL, and PA) to be used for every treatment.

17. Review the CTV1 and CTV2 treatment plans independently and in summation to ensure that they meet the dose constraints for prostate cancer irradiation established by the RTOG 0126 trial $^{19}$.

1. For the bladder volume, ensure that the percentage of tissue receiving $80 \mathrm{~Gy}$ is under $15 \%$, the percentage receiving $75 \mathrm{~Gy}$ is below $25 \%$, the percentage receiving $70 \mathrm{~Gy}$ is under $35 \%$, and the percentage receiving $65 \mathrm{~Gy}$ is less than $50 \%$.

2. For the rectal volume, ensure that the percentage of tissue receiving $75 \mathrm{~Gy}$ is below $15 \%$, the percentage receiving $70 \mathrm{~Gy}$ is under $25 \%$, the percentage receiving $65 \mathrm{~Gy}$ is less than $35 \%$, and the percentage receiving 60 Gy is below $50 \%$.

3. For the penile bulb volume, ensure that the mean dose is under $52.5 \mathrm{~Gy}$.

4. For the CTV1 and CTV2 target volumes, ensure that at least $95 \%$ of both volumes receive the prescribed dose.

18. If the plans and dose distributions meet accepted dose constraint guidelines and robustness, obtain physician approval and export the plans to the treatment delivery system.

19. Measure to confirm the accuracy of the planned dose using ionization chamber arrays, a type of radiation detector.

20. Verify the accuracy of the dose calculation using a secondary, independent dose calculation software.

21. Review the measurement results, calculation results, and technical properties of the plan with a medical physicist to ensure quality control.

22. Generate the treatment planning documents and approve them by the planning dosimetrist, physicist, and supervising physician.

23. Export all treatment planning data to the treatment delivery system for patient treatment and skip to Step 4.1 for proton therapy delivery.

\section{Radiation Treatment Planning for Passive Scatter or Uniform Scanning Proton Therapy:}

1. Import CT simulation data into the radiation treatment planning system.

2. Contour all relevant geometric volumes based on acquired CT images. These structures include the bladder, rectum, large bowel, small bowel, femoral heads, outer body surface, fiducials, rectal spacer, and/or endorectal balloon.

3. Create an additional external contour. Use the Boolean Operation tool to include the body, the treatment table, and any immobilization devices. Radiation dose will only be calculated within this contour.

4. Contour CTV1 to include the prostate, seminal vesicles, and involved lymph nodes. The CTV1 will be prescribed $45.0 \mathrm{~Gy}$ (RBE).

5. Contour CTV2 to include the prostate and seminal vesicles. The CTV2 will be prescribed a total dose of $34.2 \mathrm{~Gy}$ (RBE).

6. Expand CTV1 by $7 \mathrm{~mm}$ to create pPTV1 and create pPTV2 by expanding CTV2 by $7 \mathrm{~mm}$ in all directions except $5 \mathrm{~mm}$ posteriorly

7. Create beams on the treatment planning system to target pPTV1 and pPTV2. pPTV1 will be targeted using a single $180^{\circ}$ PA beam while pPTV2 will be targeted using $90^{\circ}$ and $270^{\circ}$ lateral beams.

8. Add blocks for each beam with a uniform margin of $0.5 \mathrm{~cm}$ to $\mathrm{pPTV} 1$ and pPTV2 volumes.

9. Using the block size select the smallest beam aperture size possible based on the size of each pPTV volume. The beam aperture is the brass custom cutout which will be attached to the gantry snout to shape lateral edges of each proton beam.

10. Model appropriate wax compensator required to shape the distal and proximal margins of each proton beam by selecting the appropriate beam parameters as follows.

1. Input a range uncertainty value of $3.5 \%$ plus an additional $1-2 \mathrm{~mm}$.

2. Input the appropriate air gap distance between the aperture and patient.

3. Smooth and smear the compensator shape to the desired dose gradient.

4. Set the isocenters for PPTV1 and pPTV2 to the same location with the goal of minimizing patient shifts required for proton beam delivery.

11. Calculate the dose using parameters entered in Steps 3.10.1-3.10.4 for both the pPTV1 and pPTV2 target plans.

12. Review the pPTV1 and pPTV2 treatment plans independently and in summation to ensure that they meet the dose constraints for prostate cancer irradiation established by the RTOG 0126 trial $^{19}$ and described in Steps 2.17.1-2.17.4.

13. If desired OAR and target goals for dose coverage are not achieved, then adjust block and compensator parameters in the TPS as shown in Steps 3.8-3.10 until the goals are achieved. Once the goals are attained, obtain physician approval and proceed to Step 3.14.

14. Verify the accuracy of the dose calculation in the approved plan using a secondary, independent dose calculation software package.

15. Review the measurement results, calculation results, and technical properties of the plan with a medical physicist to ensure quality control.

16. Order the blocks and compensators from appropriate vendor.

17. QA the blocks and compensators received from the vendor. 
18. Generate treatment planning documents and approve them via digital signatures by the planning dosimetrist, physicist, and supervising physician.

19. Export all treatment planning data to the treatment delivery system for patient treatment and proceed to Step 4.1.

\section{Radiation Treatment Delivery}

1. On the first day of treatment, check to ensure that the radiation plan matches the plan parameters in the treatment system.

2. Arrange the treatment room to reproduce the patient setup used during CT simulation. Ensure that the immobilization cushion label matches the patient ID and then place on the treatment table with correct indexing. Place the headrest utilized at simulation at the head of the table.

3. Confirm with the patient that they have completed the full bladder process and changed into a treatment gown.

4. Escort the patient into the treatment room and place him in supine position on the treatment table with hands clasped across the chest and legs in the immobilization cushion.

1. Insert and inflate endorectal balloon if used during simulation.

5. Electronically move the treatment table from the load position towards the isocenter to align the patient to the marks that are placed during simulation. Adjust the table to correct any gross errors in patient positioning such as pitch, rotation and yaw.

1. Once the patient is properly aligned to the simulation marks, complete the shifts from the start position to those determined during the dosimetric treatment planning process to align the patient to the desired treatment isocenter.

6. Perform orthogonal KV imaging to ensure proper internal patient alignment to pelvic bones and fiducial markers previously placed by urology within the prostate.

1. Determine if positional adjustments are required based on overlaying the acquired KV images on digitally reconstructed radiographs from the planning CT simulation scan. Apply necessary shifts to ensure alignment.

2. If $\mathrm{KV}$ images demonstrate excessive bowel gas, ask the patient to expel the air while lying on the treatment table if possible, then realign and re-image.

1. If the patient is unable to release on the treatment table, abort the treatment and have the patient go to the restroom. After the patient returns from the restroom, restart the proton delivery process from Step 4.3 .

7. Once acceptable KV images are acquired and confirmed, a cone beam CT (CBCT) scan is completed to assess bladder/rectal filling. Apply additional patient positioning adjustments based on the CBCT scan. As with KV imaging-based positioning corrections, changes made using СBCT data will be sent directly from the imaging console to the treatment table for automated application.

1. Verify all positional corrections with the treating physician before initiating the treatment on the first day of proton therapy.

8. Initiate the treatment delivery with audible verification between two therapists of gantry angle, monitor units, number of scanning spots and layers, and snout position for each treatment angle. These parameters are displayed on the treatment console and in the planning documents signed by dosimetry, physics, and the treating physician.

9. After the treatment, mark the treatment isocenter for daily alignment and remove the marks.

10. Repeat Steps 4.2-4.9 for all subsequent treatment fractions.

\section{Representative Results}

Available data suggests a substantial benefit with proton therapy for certain cancers ${ }^{20,21}$. PT may be favored for select pediatric tumors, recurrent cancers in previously irradiated regions, or other cancers where the risk of normal tissue injury is high with photon treatment. Below, we discuss the application and benefit of proton therapy for prostate, breast and medulloblastoma. Our aim is to provide readers with a better understanding of the application of proton therapy for tumors common in men, women, and children.

In the United States, prostate cancer is the most commonly diagnosed malignancy in men and the second most common cause of cancer-related death among men. An estimated 164,690 new cases will be diagnosed in 2018, and over 29,000 men will die of the disease. Non-metastatic prostate cancer patients are eligible for treatment options, including active surveillance, radical prostatectomy, brachytherapy and external beam radiation with photons or protons ${ }^{22}$. Exact treatment decisions are made depending on patient anatomy, comorbidities, tumor stage, physician judgment and patient preference.

Radiation delivery for early stage prostate cancer is limited to the prostate gland. In the case of intermediate risk prostate cancer, the proximal seminal vesicles are targeted as well. Although partial prostate therapies are being explored, whole gland therapy remains the standard of care. Obturator, pre-sacral, internal iliac, and external iliac nodes are often included for patients with unfavorable intermediate and high-risk disease.

Prior to radiation treatment planning, fiducial markers may be placed to permit image-guided treatment using pre-treatment kilovoltage imaging (i.e., standard X-rays $)^{23}$. In addition, a hydrogel spacer may also be inserted prior to CT simulation to create a gap between the rectum and prostate to further limit dose to the rectal tissues ${ }^{24,25}$. During treatment planning, patients should be simulated in the supine position with the pelvis immobilized using a customized cushion device. A rectal balloon may be placed at CT simulation to limit both prostate motion and uncertainty regarding rectal volume and density ${ }^{26}$. A comfortably full bladder is recommended to limit dose to the small bowel and the anterior portion of the bladder ${ }^{27}$. MRI simulation is also advised to permit more accurate target volume delineation ${ }^{26}$. 
Treatments should be designed to deliver doses of 75.6 - 79.2 Gy to the prostate, with doses of $45-50.4$ Gy recommended for elective coverage of nodal or seminal vesicle regions at risk of microscopic disease spread ${ }^{9}$. All fractions are delivered once daily in 1.8 to $2 \mathrm{~Gy}$ per fraction. For intermediate and high-risk patients receiving a brachytherapy boost, the external beam radiation dose should be limited to approximately $45 \mathrm{~Gy}$. Brachytherapy doses of 110 Gy should be used with I-125 low dose rate permanent implants. With high dose rate brachytherapy delivered via catheters, commonly used boost regimens include 13 to $15 \mathrm{~Gy} \times 1$ fraction, 8 to $11.5 \mathrm{~Gy} \times 2$ fractions, 5.5 to $6.5 \mathrm{~Gy} \times 3$ fractions, and 4.0 to 6.0 Gy $\times 4$ fractions ${ }^{9}$.

Treatment planning dosimetry is optimized to limit dose to the bladder, rectum and bowel. Dosimetric comparisons between photon- versus proton- based therapy (i.e., IMRT versus IMPT techniques) have demonstrated improved sparing of doses to normal tissues with the latter approach $^{28}$.

Prostate cancer specific mortality is under $2 \%$ at 10 years for men with early stage disease ${ }^{22}$ regardless of the treatment selected. With doseintensified RT, high risk patients also show a low prostate cancer specific mortality of $5 \%$ at 9 years ${ }^{29}$. Mortality remains low largely due to the availability of systemic therapies that remain effective in the metastatic setting. Results with both IMRT and proton therapy remain excellent ${ }^{30,31}$ The PARTIQoL (NCT01617161) study is an ongoing, randomized study between proton beam therapy (PBT) and IMRT for low and intermediate risk prostate cancer which will hopefully determine if one modality is superior over the other.

Breast cancer is the most commonly diagnosed malignancy in women and the second most common cause of cancer-related death among U.S. women. An estimated 268,670 new cases will be diagnosed in 2018 , and 41,400 women will die of the disease ${ }^{1}$. Unlike in prostate cancer where most patients receive radiation as monotherapy, breast cancer patients receive radiation postoperatively to reduce the risk of cancer recurrence ${ }^{11}$. Depending of the extent of surgery required, radiation may be targeted to the remaining breast after tumor lumpectomy or to the chest wall after mastectomy ${ }^{11,32}$. Regional lymph nodes in the axilla, supraclavicular and internal mammary areas may be targeted if they are deemed at risk for tumor spread.

Treatment schedules for breast patients typically entail once daily treatment, five days per week. Early stage patients are generally treated with conventionally fractionated (1.8-2.0 Gy/fraction; $50 \mathrm{~Gy}$ total) or hypofractionated (2.67 Gy/fraction; 40.05-42.56 Gy total) regimens to the whole breast $^{11,33}$. Patients with more advanced, but localized disease are treated with conventional fractionation to $50 \mathrm{~Gy}(1.8-2.0 \mathrm{~Gy} /$ fraction) to the whole breast or chest wall and regional lymph nodes. These doses are effective for subclinical disease which may be present following surgery.

CT simulation for breast cancer radiotherapy is typically completed in the supine position. In contrast to prostate cancer, both arms are abducted overhead to permit exposure of the chest wall or breast tissue. In addition, a customized cradling device and breast-board are often utilized to immobilize the thorax in a raised position so that the manubrium is parallel to the treatment table. This ensures that the breast tissue does not fal superiorly to the neck area.

Radiation exposure to the heart during breast cancer is associated with an increased risk of future ischemic disease ${ }^{34}$. As a result, techniques for minimizing heart doses are of paramount importance. One approach is to employ deep-inspiratory breath hold (DIBH) to increase the intrathoracic space and the distance between the heart and anterior chest wall/breast. As the method implies, patients treated with DIBH will suspend their respiratory cycle and receive treatment at the maximum point of inspiration. However, not all patients are able to tolerate breath holds of sufficient duration to permit this technique. In some patients, a prone position may be advantageous and may permit breast tissue to hang away from critical normal tissues, including the heart ${ }^{35}$. A drawback of this approach is the limitation it places on the ability to target lymphatics regions. Proton therapy can achieve substantial cardiac dose sparing without the need of $\mathrm{DIBH}$ and prone techniques ${ }^{36,37}$.

Proton therapy is employed for breast cancer patients and has been demonstrated to be superior to photon-based techniques with respect to dose sparing effects on critical structures such as the lungs and heart ${ }^{38}$. A single field pencil beam scanning (PBS) plan with a range shifter may be utilized to administer proton radiation to the chest wall and regional nodes. Passive scatter approaches may also be employed. If multiple fields are required in order to treat the entire chest wall and regional nodes due to field limitations, then field matching techniques must be employed. One strategy is to employ matching supraclavicular and chest wall fields matched with a skin gap of 2-4 mm below the clavicular head $^{39}$. The field borders are moved over a $1 \mathrm{~cm}$ distance at different time points during the radiation course to minimize hot and cold spots.

Clinical results with breast cancer radiation demonstrate an overall survival of $50 \%$ for early stage disease ${ }^{11}$ and $37 \%$ for locally advanced patients at 20 years follow-up ${ }^{32}$. Given the long remission period, minimization of treatment related toxicity is of great concern. Although proton therapy is expected to potentially lower cardiac toxicity risks, this question is being examined in the ongoing RADCOMP Consortium Trial (NCT02603341), which is randomizing women with breast cancer to photon or proton radiotherapy.

Cancer remains the second most common cause of death in children aged 1 - 14 in the United States and is only surpassed by accidents. In 2018, 10,590 children will be diagnosed with cancer, and 1,180 will die of their malignancy ${ }^{1}$. Among this group, $250-500$ patients will be diagnosed with medulloblastoma. The median age at diagnosis of medulloblastoma is $4-6$ years. Given the high risk for cerebrospinal fluid involvement and dissemination (30-40\%), craniospinal irradiation (CSI) is standard of care in these patients, with approximately $80 \%$ surviving with appropriate treatment.

Medulloblastoma patients are stratified into standard-risk and high-risk groups based on their age, presence of anaplasia or metastases, and amount of residual tumor after surgical resection. In either case, treatment includes postoperative radiation. RT for medulloblastoma involves initial CSI to a dose of 23.4 - $36 \mathrm{~Gy}$. Additional dose is then given to the tumor bed to achieve a dose of $50.4-55.8 \mathrm{~Gy}$ to the primary tumor $\mathrm{site}^{40}$. Treatment planning considerations include the limitation of the maximum doses to the brainstem and spinal cord to $54 \mathrm{~Gy}$ and $45 \mathrm{~Gy}$, respectively. CSI can be delivered using photon or proton therapy. CT simulation and treatment often require anesthesia to ensure that the patients do not move during treatment ${ }^{4}$. 
Due to the large areas targeted with radiation, photon-based RT techniques result in substantial irradiation exposure to thoracic and abdominal structures anterior to the spinal cord, including the lungs, heart, kidneys bowel, and breast. These regions may be spared from excess radiation with proton therapy (Figure 3) ${ }^{42}$. PT based CSI requires two slightly oblique lateral fields to irradiate the upper cervical spine and brain, as well as one or more posterior-anterior beams targeted to the lower cervical, thoracic, lumbar, and sacral spine regions. Multiple fields are required since the target CTV for CSI includes the entire cerebrospinal fluid (CSF) space extending from the brain vertex to the spinal canal through the cauda equina at the level of the S2/S3 vertebral junction (Figure 3). The spine length determines the total number of spinal fields required for treatment. The superior border of the uppermost spinal field is matched to the inferior border of the cranial fields. If the spine field cannot cover the entire spine, then a second spinal field is matched to the inferior border of the upper spinal field. This process may be repeated if a third field is required for taller patients. For patients below age 15, the anterior border of the spine fields is extended to include the entire vertebral bodies to ensure a homogeneous dose to bone required to prevent future growth abnormalities in the developing skeleton. For those over age 15 , the anterior spine field border is extended 2-3 $\mathrm{mm}$ beyond the spinal canal into the spinal column.

Both passive scatter and PBS techniques have been utilized for $\mathrm{CSI}^{42,43}$. Specific goals of CSI therapy include homogeneous radiation dose to the cerebrospinal fluid (CSF) to the lower end of the thecal sac (S2 or S3), full dose to the anterior skull base and cribriform plate, minimization of dose to optic structures, limitation of thyroid to no more than $5 \%$ of the prescription dose, and minimization of dose to the esophagus ${ }^{43}$.

Passive scatter treatment planning typically begins with the creation of cranial fields. Range compensators with manual editing are often required to create a homogeneous dose distribution in the brain while limiting dose to the eyes and cochlea. For spinal fields, compensators are thickened at the thyroid level to minimize dose. Special attention is then paid to field junctions between the cranial and spinal fields and between multiple spinal fields when required. The junction area is defined as the $1.25-1.5 \mathrm{~cm}$ length where the fields adjoin. The junction is shifted in the cranial or caudal direction weekly to prevent development of hot or cold dose areas. Ideally, dose variance is kept between $95-108 \%$ of the prescription dose. Field weighting, aperture edits, and compensator edits are all employed to achieve this goal ${ }^{43}$.

Researchers at M.D. Anderson Cancer Center have developed a step-wise strategy for CSI planning ${ }^{42}$. This approach involves the development of an MFO plan to treat the cranial and lower spine fields followed by the creation of an SFO plan for the thoracic spine. Dose gradients are utilized at junction areas. The SFO plan is then copied into the initial MFO plan to develop a final, composite MFO plan. Spine junctions are shifted once by $2 \mathrm{~cm}$ over a 4 -week course of treatment. In comparison to passive scatter CSI, PBS based CSI offers substantial reductions in radiation dose to the lenses, cochlea and parotid glands but at the cost of increased thyroid dose ${ }^{42}$.

Medulloblastoma patients can expect event free survival rates of $60-80 \%$ depending on the risk strata ${ }^{44}$. Given the large area of irradiated tissue with CSI, and the sensitive nature of pediatric patients, long-term side effect risks are considerable and include neurocognitive impairment, secondary malignancies, pituitary dysfunction, hearing loss, heart disease, infertility, hypothyroidism, vasculopathy, dry eyes, cataract formation, vision loss, and radiation necrosis/myelitis. Therefore, proton-based CSI may offer a substantial benefit for many patients.

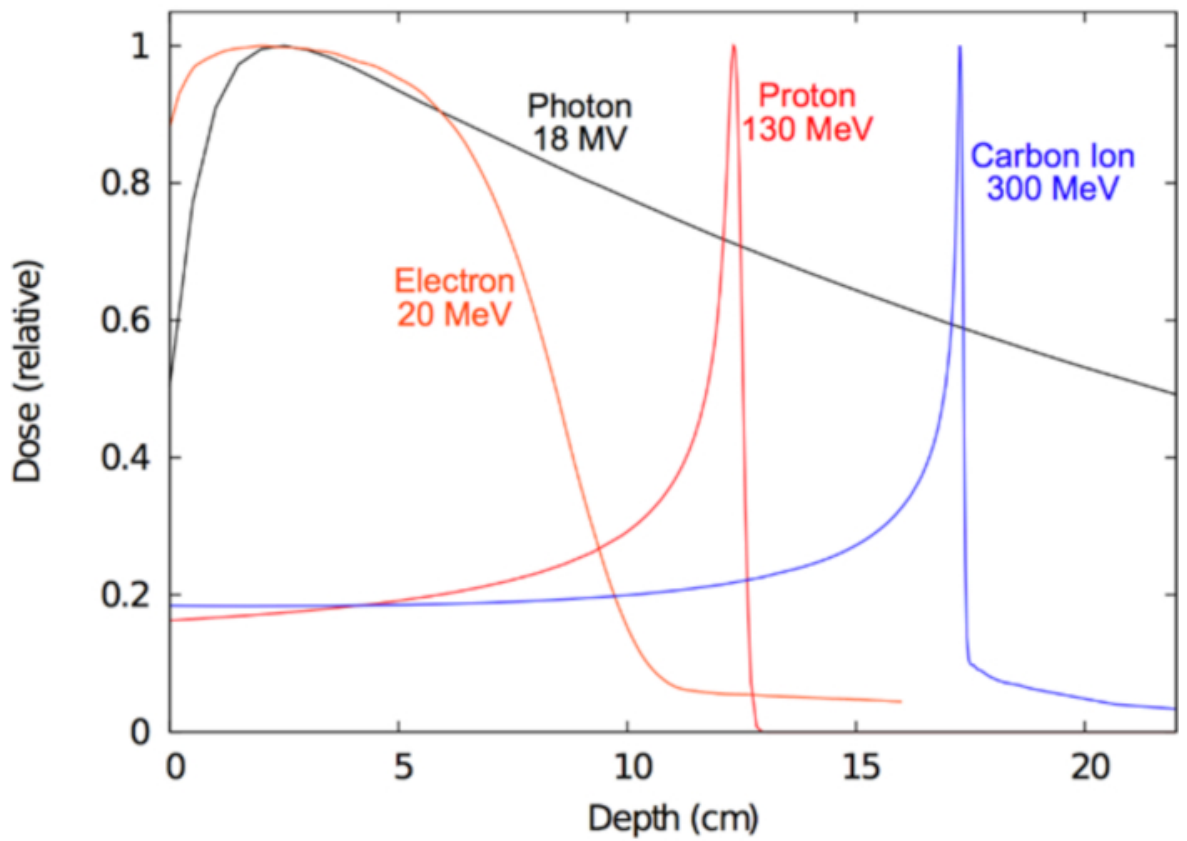

Figure 1: Depth dose curves for radiotherapy. Dose distributions as a function of depth in water shown for various clinical radiation beams. Please click here to view a larger version of this figure. 

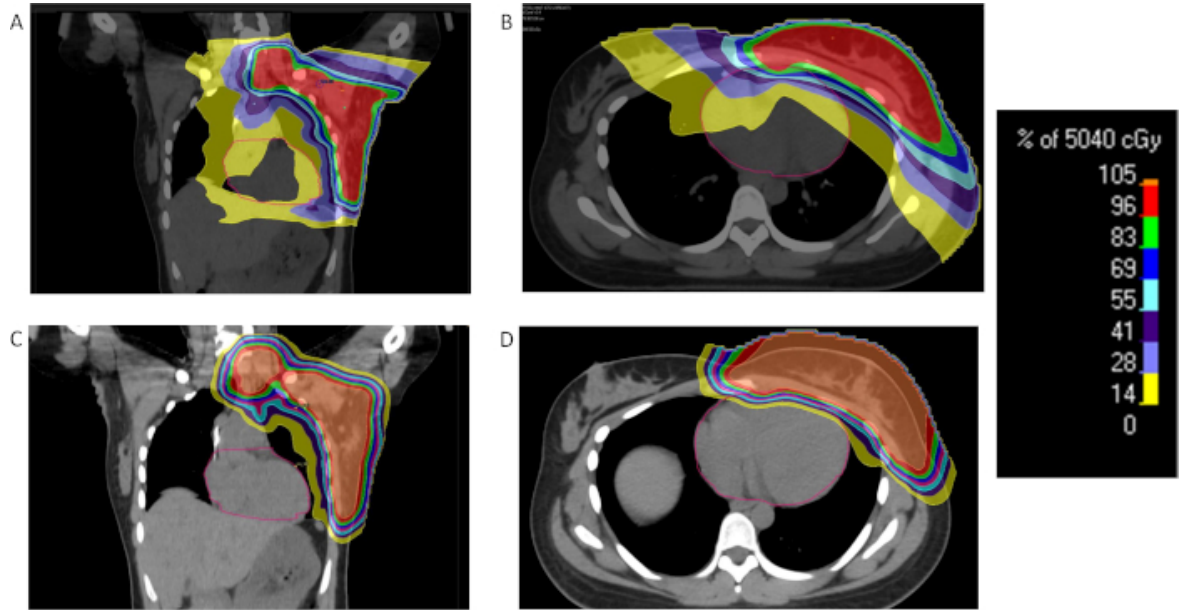

Figure 2: Comparison of proton and photon breast radiation. Percent dose distribution for a patient with locally advanced breast cancer receiving radiation therapy with either IMRT $(\mathbf{A}, \mathbf{B})$ or Protons $(\mathbf{C}, \mathbf{D})$ and demonstrating substantial radiation dose reduction to the heart and lungs with protons. Please click here to view a larger version of this figure.
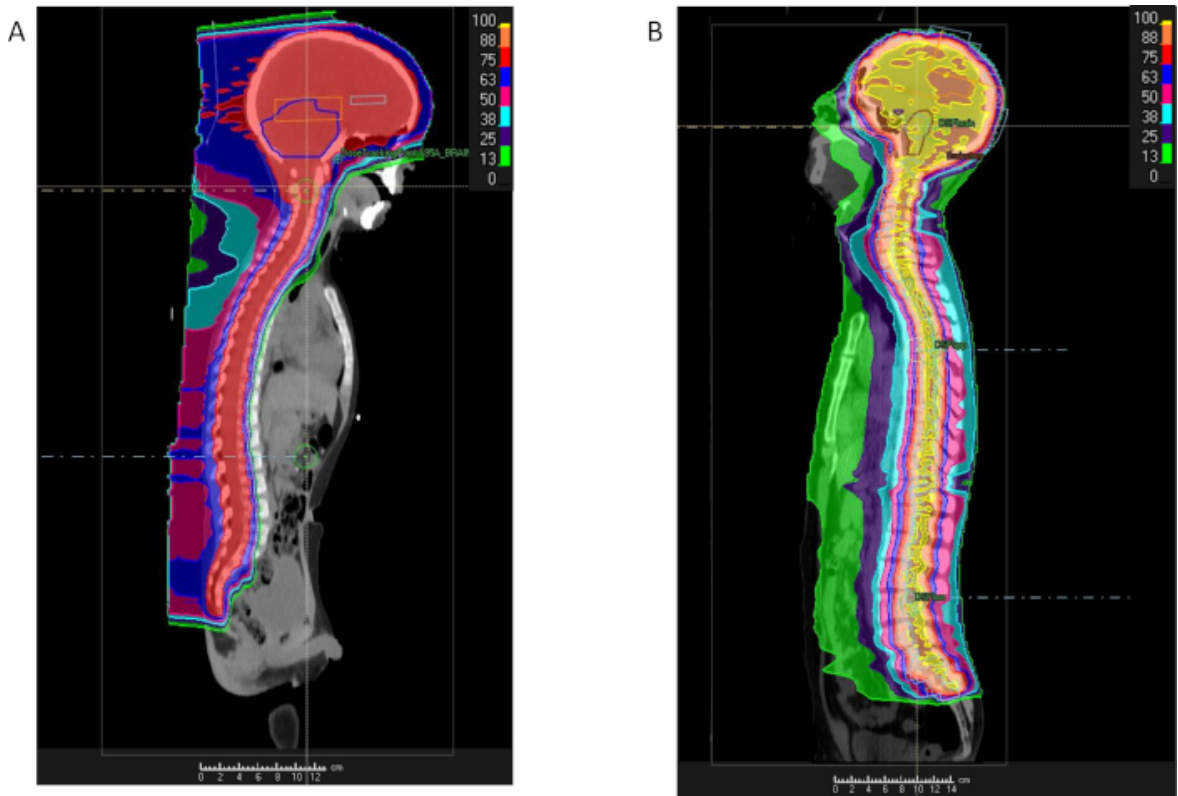

Figure 3: Comparison of proton and photon craniospinal radiation. Percent dose distribution for a patient with medulloblastoma receiving craniospinal irradiation using either Protons (A) or IMRT (B) and demonstrating substantial radiation dose reduction to intra-thoracic and intraabdominal regions with protons. Please click here to view a larger version of this figure.

\begin{tabular}{|l|l|l|}
\hline & Photon & Proton \\
\hline Particle Type & Boson & Composite Fermion \\
\hline Charge [C] & 0 & $+1.602 \times 10^{-19}$ \\
\hline Mass [kg] & 0 & $1.672 \times 10^{-27}$ \\
\hline Spin & 1 & $1 / 2$ \\
\hline Energy† [MeV] & $0.1-25$ & $10-250$ \\
\hline Common Sources & Linear Accelerator, Co-60 Radioisotopes, X-ray & Cyclotron or Synchrotron Accelerator \\
\hline Delivery Methods & Tube & Passive Scattering, Magnetic Scanning \\
\hline$\dagger$ & $\begin{array}{l}\text { Collimated Solid Beams, Multileaf Collimators, } \\
\text { Intensity Modulation, Arcs }\end{array}$ & \\
\hline
\end{tabular}

Table 1: Comparison of proton and photon radiation. 
Discussion

Radiation treatment planning and delivery for cancer is a highly customized process personalized to each individual patient and his/her particular cancer. Modern radiation treatment is an image-guided intervention-based CT images obtained during a customized radiation planning simulation. CT imaging is mandatory since it contains 3-dimensional (3D) anatomic information about the patient as well as precise quantification of the tissue densities at different locations within the body that are required for dose calculation. During CT imaging, the patient is positioned on a motorized table. Several mechanical immobilization devices are typically employed to restrict patient movement during imaging and during subsequent RT delivery. Depending on the required precision, these devices range from simple mold-type cushions and plastic meshes, which conform to the patient surface and then harden to restrict motion, to more invasive devices such as rigid skull devices that are drilled in place. Oftentimes, the required precision of the immobilization device is dictated by the proximity of the tumor tissue to nearby critical structures. As an example, the most invasive immobilization device, a head halo drilled into place, is sometimes used when single millimeter precision is needed to treat a tumor near the eyes or optic nerves to minimize the chance of blindness that may occur from the patient moving into an incorrect position during treatment.

CT imaging information is also used to optimize internal normal tissue anatomy. For example, bladder distention is often utilized to minimize bladder and small bowel dose exposure from irradiation of the prostate as noted in the protocol above. Similarly, if the stomach is notably distended with food during simulation for upper abdominal irradiation (for instance, gastric, liver, distal esophagus), then the patient is resimulated after allowing the food to pass through the stomach and intestinal tract. This will shrink the stomach and reduce the chance of radiation exposure during radiotherapy of upper abdominal tumors. In cases where the stomach or bladder themselves are radiation targets, they may be intentionally distended or emptied to optimize dose distribution.

In some instances, a tumor is not adequately or reliably visualized on CT but may be identified more accurately by an MRI or a PET scan. In such cases, the PET or MRI scans are used to complement CT data since the latter is still required for dose calculation. This is achieved by registering the MRI and PET images to CT images for planning therapy. MRI scans often provide much greater visual contrast and higher resolution than $\mathrm{CT}$, which may be beneficial to identify thin, soft-tissue boundaries of a tumor such as those in the brain or liver. PET provides a functional view of the distribution of radioactive-labeled tracer molecules injected into the patient.

Some tumors arise in areas of the thorax or abdomen where they may move significantly with respiration. In order to account for this motion to ensure radiation accuracy, a 4-dimensional CT, a type of "movie-mode" CT imaging, may be used to capture the 3D patient anatomy as it changes over time during respiration. For some thoracic and abdominal targets, compression belts or other means of motion mitigation may be used during therapy to restrict motion and limit uncertainty regarding tumor location ${ }^{45}$.

Once the patient is simulated for treatment, a personalized treatment plan is developed with consideration of the cancer histology, tumor location, and anatomic features, which influence the optimal configuration of radiation beams, particle types, energies, and dose levels for each individual patient. For each patient, a number of basic questions are initially considered by the clinical team to develop an optimal treatment plan. As a starting point, the most appropriate form of radiation must be selected. Options include photons, electrons, or protons. This is typically followed by the selection of beam angle(s) for radiation delivery. Most RT machines include a robotic patient positioning table and a rotating gantry that allow RT beams to be directed into the patient by virtually any angle. The decision involves finding the path that most effectively strikes the target with RT and best avoids non-targets that may be in the path of the selected beams. In some cases, beam angles are determined by the planning system itself after inputting in radiation goals for tumors and normal tissues. This process is termed "inverse planning" and is often done in the case of IMRT, which involves modulating the intensity of multiple, incoming radiation beams in a time-dependent manner that provides a uniform target dose but may lead to highly non-uniform dose outside the target. Although both photon or proton therapy may be intensity modulated, inverse planning is utilized largely in photon based IMRT only. If solid radiation beams are to be used, custom metal collimators may be fabricated to match the shape of the radiation beam with the shape of the tumor.

If proton therapy is selected, then a subsequent decision needs to be made regarding the use of passive scatter or PBS techniques. In the case of PBS, an additional decision is required regarding the use of MFO or single-field optimization/single-field uniform dose (SFO/SFUD) strategies. In MFO treatments, multiple beams are required to treat a tumor during every fraction since each beam only targets a portion of the target. In contrast, for SFO plans, every beam covers the entire target. MFO is often favored for tumors near to a critical structure (e.g., brain tumor near the optic nerve) where a variety of beam angles may be advantageous to sculpt radiation dose. MFO strategies also ensure that all the radiation beams/spots do not "end range" into the same area where dose could be unexpectedly high due to the Bragg Peak effect. On the other hand, SFO is favored for targets near areas of anatomic uncertainty, such as the prostate which can move due to differential bladder and rectal filling. SFO provides enhanced robustness against dose changes due to anatomic variances.

Once the basic planning strategy is decided, the next phase of treatment planning typically involves mathematical optimization of the radiation fields. The energy, intensity, and spatial distribution (spatially varying flux) of incoming radiation are typically free parameters in optimization. Along with the large 3D matrix representation of the patient anatomy by $\mathrm{CT}$, these free variables lead to a very large problem size and corresponding large optimization matrices (e.g., thousands of CT values and thousands of possible beam intensities must be considered). These matrices are framed in an Objective Function, which is a mathematical formulation of the "goal of treatment planning". As mentioned above, treatment goals are prioritized to first achieve the prescribed dose to the target, and secondly to achieve as low of a dose as is possible to normal tissues. To minimize this Objective Function, high computing power is desired to quickly perform RT transport calculations that populate the matrices, and numerical optimization methods, such as gradient-search algorithms, are used to quickly search for local minima in the function. These minima correspond to optimal treatment plans for each unique patient. The role of computers in treatment planning cannot be overstated. Modern radiation treatment and diagnostic radiology would not be possible without the computer advancements of the past three decades. 
As a final stage, the optimized treatment plan is reviewed by the medical team (physician, dosimetrist, and physicist). In many cases, the plan may be adapted further or re-optimized with differing objectives to improve overall quality. Once the plan is found to be optimal, the technical parameters of the plan are reviewed by a physicist and transferred to the treatment delivery machine.

In many cases, the patient returns for multiple treatment fractions (sessions), often every weekday for several weeks. Multi-day fractionation can intensify acute radiation-induced side effects but may reduce potential late, more severe side effects of RT compared with single-fraction treatment $^{12}$. Multi-fraction approaches are optimal for tumors that are rapidly dividing or unable to repair sublethal damage from RT. However, this depends on the exact treatment site and the sensitivity of the nearby normal tissues. Since the goal of radiation treatment delivery is to administer the same treatment during each fraction, even a few millimeters of motion or uncertainty in the patient position may lead to the degradation of the particle therapy treatment plan. For this reason, on-board image guidance systems are of paramount importance during multifraction RT. X-ray imagers, cone beam CT scans, or optical, laser-scanning surface imagers are all available for this purpose. These devices permit image-guided radiotherapy (IGRT) through the imaging of anatomical landmarks, tumor targets, or surrogate radio-opaque fiducial markers. The IGRT images are compared to the original simulation scans and adjusted as necessary prior to each fraction of radiation.

Despite the advantage of the finite range of proton therapy, which limits the exit dose, the precision of range prediction typically seen in treatment planning is on the order of a few millimeters. The exact energy loss in different patient tissues is uncertain, firstly, since the exact molecular components of the tissue are ambiguous, and, secondly, since the patient anatomy changes over time, both over short timescales (e.g., breathing) and longer timescales (e.g., weight loss, tumor shrinkage, normal anatomy changes). To address this uncertainty, a "distal margin" is added to the target volume that is an additional margin of normal tissue just beyond the maximum tumor depth. Such a margin ensures that even with the uncertainties in range prediction, the entire tumor depth will be treated with high confidence. Unfortunately, the normal tissue margin can as a result be exposed to the full RT dose, which can potentially lead to significant RT side effects in that tissue. In contrast, as photons do not stop but rather exit the target, no such distal margin is needed to compensate range uncertainty. A geometric margin is still used in photon therapy to address positional uncertainties of the target, but photons are much less sensitive than protons to the precise status of patient tissues upstream of the target. Therefore, the needed margin can sometimes be smaller for photons than protons. This can be understood by considering that protons undergo continuous energy loss in tissues that greatly affect the position of their range, whereas photons are uncharged and travel freely in the empty space between atoms and their orbitals, except for rare collisions with electrons or nuclei. Large density differences in tissue, e.g., metallic objects or air cavities, however, still influence photon dose as well as proton dose, but to a lower magnitude.

A final and important uncertainty pertains to the radiobiological effectiveness (RBE) of different forms of radiation. The RBE is the ratio of doses, from a reference radiation type and a test radiation type, under the condition that both radiation types produce the same biological effect. The higher the RBE, the more damaging the radiation per unit of energy deposition in tissue. The RBE ratio is defined in reference to photon radiation. Despite this straightforward description, there is actually great uncertainty regarding the RBE values for charged particles as opposed to photons. Differences in the spatial dose distributions between photons and charged particles at the micrometer and nanometer scale lead to differences in biologic effect, even when the macroscopic doses are identical. This can be understood by examining the spatial patterns of DNA damage after exposure to charged particles at different doses and different kinetic energies. Different kinetic energies and different charges of protons $(+1)$ and carbon ions $(+6)$ lead to the differences in energy transfer at different depths in the patient, whereas for photons, the energy transfer is comparably lower and also more homogeneous throughout the patient. While theoretically understood, there is significant debate in the radiation oncology community regarding the ability to accurately predict such biologic effects. For carbon ion therapy, there is a lack of consensus on how best to model these biologic effects, though there is agreement that such effects must be modeled to provide therapy. For protons, most clinical centers currently plan therapy without explicit modeling of RBE effects, except for using a constant correction factor of 1.1 , but this is likely to change in the near future as new commercial treatment planning systems are beginning to include biological modeling software tools to model the RBE of proton therapy.

With the completion of randomized trials, including RADCOMP, PARTIQoL and RTOG 1308, we should have more concrete answers as to which forms of radiation may be superior for breast, prostate, and lung cancer, respectively. Similar studies are planned for other disease sites that may help to better identify the best treatment modality for those tumor types. However, there is already sufficient data to suggest the superiority of protons in certain settings, particularly in the pediatric population, where substantial normal tissue sparing can greatly reduce morbidity from toxicities, including secondary malignancies.

\section{Disclosures}

A.H. has honoraria from Astrazeneca, Bayer, and Novartis. A.H. has consulted with Astrazeneca, Bristol-Myers Squibb, and Bayer and was a speaker for the France Foundation.

\section{Acknowledgments}

S.R. acknowledges grant funding from the NIH Loan Repayment Program. A.H. has received funding from Bayer, Clovis, Constellation, Agensys, Sotio, Cerulean, and Calithera.

\section{References}

1. Siegel, R. L., Miller, K. D., \& Jemal, A. Cancer statistics, 2018. CA A Cancer Journal for Clinicians. 68 (1), 7-30 (2018)

2. Barton, M. B. et al. Estimating the demand for radiotherapy from the evidence: a review of changes from 2003 to 2012 . Radiotherapy and Oncology. 112 (1), 140-144 (2014).

3. Pan, H. Y. et al. Supply and Demand for Radiation Oncology in the United States: Updated Projections for 2015 to 2025 . International Journal of Radiation Oncology Biology and Physics. 96 (3), 493-500 (2016).

4. Reed, A. B. The history of radiation use in medicine. Journal of Vascular Surgery. 53 (1 Suppl), 3s-5s (2011)

5. T Landsberg, P. Nobel Lectures in Physics, 1901-1921. Vol. 18 (1967). 
6. Non-small cell lung cancer. <https://www.nccn.org/professionals/physician_gls/pdf/nscl.pdf> (2018).

7. Pancreatic Adenocarcinoma. <https://www.nccn.org/professionals/physician_gls/pdf/pancreatic.pdf> (2017).

8. Breast cancer. <https://www.nccn.org/professionals/physician_gls/pdf/breast.pdf> (2017).

9. Prostate Cancer. <https://www.nccn.org/professionals/physician_gls/pdf/prostate.pdf> (2017).

10. Chang, J. Y. et al. Stereotactic ablative radiotherapy versus lobectomy for operable stage I non-small-cell lung cancer: a pooled analysis of two randomised trials. Lancet Oncology. 16 (6), 630-637 (2015).

11. Fisher, B. et al. Twenty-year follow-up of a randomized trial comparing total mastectomy, lumpectomy, and lumpectomy plus irradiation for the treatment of invasive breast cancer. The New England Journal of Medicine. 347 (16), 1233-1241 (2002).

12. Hall, E. J., \& Giaccia, A. J. Radiobiology for the radiologist. Wolters Kluwer Health/Lippincott Williams \& Wilkins (2012).

13. Lomax, A. J. Charged particle therapy: the physics of interaction. The Cancer Journal. 15 (4), 285-291 (2009).

14. Rossi, B., \& Greisen, K. Cosmic-Ray Theory. Reviews of Modern Physics. 13 (4), 240-309 (1941).

15. Blakely, E. A., \& Chang, P. Y. Biology of charged particles. The Cancer Journal. 15 (4), 271-284 (2009).

16. Schardt, D., Elsässer, T., \& Schulz-Ertner, D. Heavy-ion tumor therapy: Physical and radiobiological benefits. Reviews of Modern Physics. 82 (1), 383-425 (2010).

17. Chun, S. G. et al. The Potential of Heavy-Ion Therapy to Improve Outcomes for Locally Advanced Non-Small Cell Lung Cancer. Frontiers in Oncology. 7 (201), 1-3 (2017).

18. Pan, H. Y., Jiang, J., Shih, Y. T., \& Smith, B. D. Adoption of Radiation Technology Among Privately Insured Nonelderly Patients With Cancer in the United States, 2008 to 2014: A Claims-Based Analysis. Journal of the American College of Radiology. 14 (8), 1027-1033.e1022 (2017).

19. Michalski, J. M. et al. Effect of Standard vs Dose-Escalated Radiation Therapy for Patients With Intermediate-Risk Prostate Cancer: The NRG Oncology RTOG 0126 Randomized Clinical Trial. JAMA Oncology. (2018).

20. Glimelius, B. et al. Number of patients potentially eligible for proton therapy. Acta Oncologica. 44 (8), $836-849$ (2005).

21. Doyen, J., Falk, A. T., Floquet, V., Herault, J., \& Hannoun-Levi, J. M. Proton beams in cancer treatments: Clinical outcomes and dosimetric comparisons with photon therapy. Cancer Treatment Reviews. 43, 104-112 (2016).

22. Hamdy, F. C. et al. 10-Year Outcomes after Monitoring, Surgery, or Radiotherapy for Localized Prostate Cancer. New England Journal of Medicine. 375 (15), 1415-1424 (2016).

23. Ng, M. et al. Fiducial markers and spacers in prostate radiotherapy: current applications. British Journal of Urology International. 113 Suppl 2, 13-20 (2014).

24. Hedrick, S. G. et al. A comparison between hydrogel spacer and endorectal balloon: An analysis of intrafraction prostate motion during proton therapy. Journal of Applied Clinical Medical Physics. 18 (2), 106-112 (2017).

25. Hamstra, D. A. et al. Continued Benefit to Rectal Separation for Prostate Radiation Therapy: Final Results of a Phase III Trial. International Journal of Radiation Oncology Biology Physics. 97 (5), 976-985 (2017).

26. Wortel, R. C. et al. Local Protocol Variations for Image Guided Radiation Therapy in the Multicenter Dutch Hypofractionation (HYPRO) Trial: Impact of Rectal Balloon and MRI Delineation on Anorectal Dose and Gastrointestinal Toxicity Levels. International Journal of Radiation Oncology Biology Physics. 99 (5), 1243-1252 (2017).

27. Chen, Z., Yang, Z., Wang, J., \& Hu, W. Dosimetric impact of different bladder and rectum filling during prostate cancer radiotherapy. Radiation Oncology. 11, 103 (2016).

28. Rana, S. et al. Dosimetric and radiobiological impact of intensity modulated proton therapy and RapidArc planning for high-risk prostate cancer with seminal vesicles. Journal of Medical Radiation Sciences. 64 (1), 18-24 (2017).

29. Rodda, S. et al. ASCENDE-RT: An Analysis of Treatment-Related Morbidity for a Randomized Trial Comparing a Low-Dose-Rate Brachytherapy Boost with a Dose-Escalated External Beam Boost for High- and Intermediate-Risk Prostate Cancer. International Journal of Radiation Oncology Biology Physics. 98 (2), 286-295 (2017).

30. Zietman, A. L. et al. Randomized trial comparing conventional-dose with high-dose conformal radiation therapy in early-stage adenocarcinoma of the prostate: long-term results from proton radiation oncology group/american college of radiology 95-09. Journal of Clinical Oncology. 28 (7), 1106-1111 (2010).

31. Al-Mamgani, A., Heemsbergen, W. D., Peeters, S. T., \& Lebesque, J. V. Role of intensity-modulated radiotherapy in reducing toxicity in dose escalation for localized prostate cancer. International Journal of Radiation Oncology Biology Physics. 73 (3), 685-691 (2009).

32. Ragaz, J. et al. Locoregional radiation therapy in patients with high-risk breast cancer receiving adjuvant chemotherapy: 20 -year results of the British Columbia randomized trial. Journal of the National Cancer Institute. 97 (2), 116-126 (2005).

33. Whelan, T. J. et al. Long-term results of hypofractionated radiation therapy for breast cancer. New England Journal of Medicine. 362 (6), 513-520 (2010).

34. Darby, S. C. et al. Risk of ischemic heart disease in women after radiotherapy for breast cancer. New England Journal of Medicine. 368 (11), 987-998 (2013).

35. Wroe, A. J., Bush, D. A., Schulte, R. W., \& Slater, J. D. Clinical immobilization techniques for proton therapy. Technology in Cancer Research and Treatment. 14 (1), 71-79 (2015).

36. Shah, C. et al. Cardiac dose sparing and avoidance techniques in breast cancer radiotherapy. Radiotherapy and Oncology. 112 (1), 9-16 (2014).

37. Patel, S. A. et al. Postmastectomy radiation therapy technique and cardiopulmonary sparing: A dosimetric comparative analysis between photons and protons with free breathing versus deep inspiration breath hold. Practical Radiation Oncology. 7 (6), e377-e384 (2017).

38. Depauw, N. et al. A novel approach to postmastectomy radiation therapy using scanned proton beams. International Journal of Radiation Oncology Biology Physics. 91 (2), 427-434 (2015).

39. MacDonald, S. M. et al. Proton therapy for breast cancer after mastectomy: early outcomes of a prospective clinical trial. International Journal of Radiation Oncology Biology Physics. 86 (3), 484-490 (2013).

40. Merchant, T. E. et al. Multi-institution prospective trial of reduced-dose craniospinal irradiation (23.4 Gy) followed by conformal posterior fossa (36 Gy) and primary site irradiation (55.8 Gy) and dose-intensive chemotherapy for average-risk medulloblastoma. International Journal of Radiation Oncology Biology Physics. 70 (3), $782-787$ (2008).

41. McMullen, K. P., Hanson, T., Bratton, J., \& Johnstone, P. A. Parameters of anesthesia/sedation in children receiving radiotherapy. Radiation Oncology. 10, 65 (2015).

42. Stoker, J. B. et al. Intensity modulated proton therapy for craniospinal irradiation: organ-at-risk exposure and a low-gradient junctioning technique. International Journal of Radiation Oncology Biology Physics. 90 (3), 637-644 (2014). 
43. Giebeler, A. et al. Standardized treatment planning methodology for passively scattered proton craniospinal irradiation. Radiation Oncology. 8, 32 (2013).

44. Gajjar, A. et al. Risk-adapted craniospinal radiotherapy followed by high-dose chemotherapy and stem-cell rescue in children with newly diagnosed medulloblastoma (St Jude Medulloblastoma-96): long-term results from a prospective, multicentre trial. Lancet Oncology. 7 (10), 813-820 (2006).

45. Lin, L. et al. Evaluation of motion mitigation using abdominal compression in the clinical implementation of pencil beam scanning proton therapy of liver tumors. Medical Physics. 44 (2), 703-712 (2017). 